\title{
Remembering to learn: the overlooked role of remembrance in safety improvement
}

\section{Abstract}

A fundamental objective of safety improvement is to remember and learn from the past in order to improve the future. Many patient safety improvement strategies are organised around efforts to create and maintain shared memories of past events, but healthcare systems still routinely suffer from acute episodes of forgetfulness and continue to repeat the same harmful incidents. One route to tackling healthcare's memory problems may be through the detailed analysis and careful exploration of the social practices of remembrance. Remembrance has, at its core, the purpose of collective memory-making and the maintenance of socially shared memories. Many of the social functions of remembrance would seem centrally important to patient safety. These include publicly acknowledging harm, collectively reflecting on past events, creating a shared sense of responsibility, reaffirming cultural values and priorities, emotionally engaging with suffering, and creating a space for compassion and ethical behaviour. A more detailed and systematic understanding of remembrance may therefore illuminate some fundamental aspects of organisational memory that have remained largely unexplored in patient safety. Specifically, these are the symbolic, emotive, practical, ethical and collective aspects of remembering and learning from past events. As such, the theoretical and practical implications of remembrance seem worthy of careful and systematic attention and may lead to the creation of more compassionate, reflective and just cultures of safety. 


\section{Remembering to learn: the overlooked role of remembrance in safety improvement}

Memory, and remembering the past, are fundamental to patient safety. One of the core objectives of safety improvement is to learn from the past in order to improve the future. This commitment to remember and to learn is central to the strategies that have shaped the evolution of patient safety such as "An organisation with a memory",[1] and underpins definitive academic research such as Bosk's "Forgive and Remember".[2] Remembering the past to improve the future is institutionalised across healthcare in a variety of activities such as safety incident reporting, morbidity and mortality meetings, coroner investigations and public inquiries. Despite this, healthcare systems still suffer striking and acute episodes of forgetfulness[3] that are deeply consequential: when harmful events are forgotten, they are likely to be repeated.

Given the central importance of memory in patient safety it is surprising that one of the most long standing forms of collective memory-making has remained largely unexplored in healthcare: the social practices of remembrance. These practices colour our daily lives, from memorial services that commemorate the lives of those who have passed away, to remembrance events that bring people together to remember harmful past events and honour those affected. The nature of remembrance is the subject of a small and sophisticated literature, $[4,5,6]$ but there has been little systematic exploration of these ideas in safety-critical settings in general, or healthcare in particular. However, what we already know about 
remembrance suggests that more detailed study could offer rich insights into the practical, social and emotional aspects of remembering and learning from past adverse events in healthcare. To explore these issues, this paper first examines what remembrance is and what its defining features look like, drawing on three practical examples in safety-critical settings. Then, the paper analyses the functions and purposes that remembrance might serve in organisational settings and why these resonate with current challenges in patient safety. The paper then considers the potential risks of remembrance, and concludes by considering how this new arena of research and practice might be taken forward.

\section{THE NATURE OF REMEMBRANCE}

What is remembrance and what does it look like in safety-critical settings? This question has received little empirical or theoretical attention, though the nature of remembrance has received critical attention more generally.[4,5,6] Simply, remembrance is the creation of a "space and context for the sharing of memories".[4] Remembrance, commemoration and memorialisation represent a range of social activities that share the goal of creating and sustaining collective memories of the past, and making those memories meaningful in the present. While remembrance remains under-explored in safety-critical settings, three high-profile examples illustrate how remembrance can be incorporated into efforts to improve safety (Box 1). These include permanent museums that publicly commemorate past accidents and engage and educate staff.[7] They include prestigious awards that promote improvement activities in honour of patients who lost their lives to catastrophic 
error.[8] And they include days of remembrance that bring together entire organisations to reflect on past tragedies and current implications.[9]

Remembrance remains under-theorised in safety-critical settings, but broader analyses have highlighted the centrality of four distinct features.[4] First, remembrance is practical. It is a set of activities that people actively participate in, often involving communal ceremonies or rituals. It is through engaging in practical activities that past events are made relevant and meaningful to the present. Static objects such as monuments or archives are important, but if these objects are not surrounded by some set of structured practices then memories and meaning can fade. Second, remembrance is symbolic. It involves creating and maintaining meaningful images, experiences, knowledge and artefacts of past events. These symbols make past events available to be reflected upon and explored from the vantage point of the present. Third, remembrance is emotive. It allows people to encounter the harm and trauma of past events, as well as engage with positive experiences that might have followed. Moving and emotionally affecting stories bring experiences of the past vividly into the present. Fourth, remembrance is social. It is performed together, in groups and communities, through collective and public acts. The inherently social nature of remembrance underpins its purpose of maintaining collective memories. Collective memories are those that are shared amongst a group, and form a defining feature of a culture or community. Emotions, too, are made collective through remembrance-moving from my sorrow, or your hope, to our shared sorrows and hopes.[4] 
Remembrance encompasses a diverse range of activities where the prime purpose is maintaining collective memories of past events through practical participation, symbolic meaning, emotional engagement and social connection. This can include ceremonies, museums and prizes, such as the examples in Box 1. It might also include, in healthcare, recounting patient stories at board meetings, running conferences that explore and honour the experiences of patients and families, or sharing moving narratives of adverse events through films and other media-if those activities are primarily concerned with reflecting on the past in emotionally engaging, socially situated, symbolically meaningful and participative ways. In contrast, activities that lack these characteristics-such as a cursory 'tick box' reading of a patient story, the existence of a commemorative plaque or an isolated individual thinking about historic events—could not be considered remembrance. 


\section{BOX 1: Examples of remembrance in safety-critical contexts}

\section{Museums of commemoration: J apan Airlines}

In August 1985, a J apan Airlines jumbo jet crashed west of Tokyo, 32 minutes after the rear of the aircraft was ripped away by the explosive failure of a faulty repair made seven years earlier. 520 people lost their lives in the most deadly singleaircraft accident in history. This accident is commemorated in the J apan Airlines Safety Promotion Centre.[7] This museum of aviation safety opened in 2006 and displays reconstructed wreckage —including the failed repair work-alongside destroyed seats and heart-rending messages written by passengers to their loved ones as the crippled aircraft circled in its final minutes. Another major Japanese airline, All Nippon Airways, has its own safety museum nearby. All staff are required to visit and these museums provide powerful and moving experiences that emphasise the causes and terrible consequences of failure in these organisations, and provide public and highly visible commemorations to honour those who lost their lives. Airline staff are encouraged to share their personal safety pledges, which are displayed within a library of safety improvements and examples of how specific lessons have been learnt from past failures.

\section{Days of remembrance: NASA}

Each year on J anuary 28th the US National Aeronautics and Space Administration (NASA) holds a day of remembrance for those who have lost their lives in space programme accidents. The date, J anuary 28th, is the anniversary of the 1986 Challenger shuttle accident in which seven crew died when a booster rocket exploded during launch, destroying the shuttle. The day also honours the seven crew lost on the Columbia shuttle in 2003, when the heat shield of a wing was damaged during launch and failed catastrophically during re-entry, and three crew of Apollo 13, who died in a fire on the launch pad during a test of their Apollo capsule in 1967. This day of remembrance has become a public day of reflection and remembrance, with statements made by the US President and the head of NASA, and a moment of silence is marked across the organisation and broadcast live, including via video link from the space station. Directors pay tribute to those who lost their lives, reflect on the hundreds of safety improvements that these events led to and describe how the events reaffirm the value and importance of the work being done. Videos explaining the events and related materials and accident investigation reports are posted on a dedicated website[8] and in a permanent memorial at the Kennedy Space Center. 


\section{Awards of memorial: Virginia Mason}

On November 23rd 2004, Mrs Mary L MoClinton died after she was inadvertently injected with chlorhexidine during treatment for a brain aneurism at Virginia Mason Medical Centre. This tragic event has been heralded as a turning point for transparency and patient safety at Virginia Mason, both in the immediate aftermath of the tragedy and in the long term. The hospital leaders took steps considered at the time to be highly unusual in healthcare, by openly disclosing the event and making patient safety improvement the overriding organisational priority for several years. One year on from the event, a day of memorial was held with the family and all staff, and a day continues to be set aside each year for reflection on improvement in Mrs McClinton's honour. Virginia Mason went on to establish the annual Mary L McClinton Patient Safety Award to recognise improvement work that has had the greatest impact on patients, been spread widely and sustained over time. This award is regarded as highly prestigious and meaningful within the healthcare system, and so both honours the memory of Mrs McClinton and uses this terrible event as a motivation for groups across the healthcare system to work to improve safety. This is memorialised on a public website.[9] 


\section{THE FUNCTIONS AND RELEVANCE OF REMEMBRANCE}

Remembrance serves a range of social functions that would seem directly relevant to many of the most pressing challenges faced in patient safety. While there have been no empirical studies of remembrance in safety improvement, either in healthcare or elsewhere, three areas stand out. First is the role of remembrance in ensuring that healthcare organisations openly and honestly acknowledge harmful events. Second is the role of remembrance in creating protected time and space to reflect on the wideranging causes and serious consequences of harmful events. Third is the role of remembrance in transforming harmful and tragic events into a motivating driver for positive action and ethical behaviour.

\section{Public honesty and collective responsibility}

Remembrance provides a variety of ways for organisations to publicly acknowledge harm caused by safety failings, to honour those affected and to demonstrate and model open disclosure. In so doing, organisations can visibly demonstrate a public commitment to honesty and openness. This public honesty is the antithesis of efforts to deny, cover up or ignore safety problems in ways that can cause further distress to patients and families.[10,11] A remembrance activity, such as a safety prize, may focus on commemorating a specific event, but it can also reinforce a more general and difficult message: that healthcare systems and professionals can sometimes harm those they seek to help. 
More subtly, remembrance can help create a sense of shared responsibility for safety, in ways that are entirely separate from legalistic attempts to allocate—or deflectblame. Harm in healthcare is typically individualised and localised. Adverse incidents usually harm one patient at a time, creating deeply personalised tragedies. And it can be one individual healthcare worker who carries much of the shame and stigma associated with 'causing' a harmful event. However, safety incidents are always the result of complex networks of actions and conditions, often spread across multiple organisations.[12] By bringing people together to collectively engage with the diverse causes and effects of harm, remembrance may help develop a sense of communal ownership of-and obligation for-safety improvement.

\section{Reflective inquiry and cultural identity}

Remembrance seeks to maintain memories of the past and make the past relevant to the present. One of the most instrumental functions of remembrance could therefore be to provide a context for talking about and inquiring into the precursors of safety failures, so that those precursors might be better recognised and addressed in future. Ongoing conversations about safety, including the wide circulation of memorable stories, are some of the key mechanisms through which organisations make sense of the risks they face.[13,14] By encouraging collective reflection on specific major safety events, remembrance may provide a way of maintaining shared awareness of certain types of major risk-such as high-risk medication errors. 
At the same time, remembrance could provide a space for exploring how seemingly trivial mishaps or minor issues can escalate in unexpected and devastating ways, and bring attention to the importance and impact of ostensibly lower-impact or more routine problems - such as missing equipment, delayed test results or catheterassociated infections-that are common in many healthcare settings. Relatedly, remembrance may offer a practical way of shaping the cultural identity of an organisation. Remembrance is symbolic and value-laden: honouring and remembering past events provides strong markers of what a community should take seriously, what is worthy of attention and what it should avoid. A significant opportunity for remembrance in healthcare may therefore be to contribute to a culture of wary precaution, forestalling the process of "forgetting to be afraid".[12]

\section{Emotion, compassion and ethics}

Remembrance could be useful in healthcare to provide a space in which to emotionally engage with the suffering associated with harmful events. In healthcare this may be the emotional pain and grief of those harmed or bereaved, $[15,16]$ as well as the feelings of shame and stigma of healthcare professionals.[17] Bringing emotion in to patient safety is important.[18] Emotional events create stronger and more robust memories.[19] Personal and moving stories can make the past real and meaningful to the present[4]. Negative emotion can be a strong trigger for sensemaking and collective inquiry.[20] And social expressions of anxiety and 
concern can spread,[21] supporting organisational cultures organised around the right kind of fear: of inadvertently causing harm to patients.[22]

Remembrance can also demonstrate ethical and honourable behaviour, indicating that an organisation takes seriously the ideals of truthfulness, compassion and learning. Forgetting or denying harm has a moral dimension. It suggests that suffering, and those who have suffered, are unimportant. By publicly demonstrating compassion and respect, remembrance could provide a model for more ethical responses to harmful events, contributing to more compassionate cultures of care.

\section{THE RISKS OF REMEMBRANCE}

Instituting activities of remembrance in healthcare may offer a range of opportunities to positively shape culture and support improvement. It also presents a number of risks. How these impacts might be realised, and how these risks might be mitigated, are ultimately empirical questions to be answered through close study and careful testing. The key risks of remembrance concern the focus of any remembrance activities, and the threats posed by bureaucratisation.

\section{Focus and scale}


One of the key challenges-and risks-facing remembrance in patient safety is determining how, when and where remembrance might be instituted. In the few practical examples that exist (Box 1), safety-critical organisations in healthcare and elsewhere have focused on commemorating a small number of seriously harmful events, in a defined place (such as a museum or exhibition) or moment (such as an annual award or remembrance day). If remembrance were to be instituted more widely in healthcare systems, an increased focus on a small number of rare catastrophic events might risk distracting attention from the wide variety of patient safety issues that do not routinely lead to catastrophic outcomes, such as pressure ulcers, as well as distracting attention from other serious adverse events that are not actively memorialised.

A related challenge would be determining which, and how many, adverse events to memorialise. In some industries, harmful events are rare and can harm large numbers of people. In healthcare, harm is individualised and relatively common. It would be neither practical nor appropriate to commemorate every single patient safety incident. Too great a focus on too many events risks undermining the very purpose of remembrance: highly localised events may not be perceived as meaningful or engaging for a wider community, and efforts to institute too many individual commemorations could lead to emotional fatigue and disengagement. Ultimately, issues regarding the appropriate type, size, frequency and focus of remembrance are empirical questions, but it would seem likely that appropriate activities should be chosen and scaled to support the requirements of being 
emotionally resonant, practically engaging, symbolically meaningful and socially participative within the organisation concerned.

\section{Bureaucratisation and appropriation}

Some of the most serious risks of remembrance are that attempts to honour and emotionally engage with the past can become bureaucratised, degrading into empty rituals that preclude rather than promote reflective inquiry and organisational change. Responses to major disasters that create empty procedural rituals are well documented[23] and this 'tombstone effect', where organisational routines become ossified and limit the scope of future adaptation, represent a considerable threat to improvement.[24] In healthcare, well-meaning attempts to honour past harms and provoke reflection-such as sharing patient stories at board meetings-have in places already become little more than formulaic exercises, rather than the deeply impacting and meaningful experiences they can be. The essence of remembrance is active reflection, emotional engagement and mindful participation. Without these, rituals of remembrance are simply mindless routines, and any potential benefits are lost. Of equal concern is that any institutional efforts to remember the past faces the risk of misappropriating and misusing the experiences of those harmed. Organisations may use activities of remembrance to create narratives of redemption and improvement where none exist, or seek to passively deflect or actively reallocate blame. These risks confront any organised attempt to understand harm, and 
represent distortions of the act of collective remembering intended to shut down rather than support the meaningful exploration of the past.

\section{THE REMEMBRANCE OF HARMS PAST}

Many patient safety activities are based on memory-making activities, but healthcare still routinely struggles to meet the practical demands and moral obligations of remembering the past to improve the future. If our healthcare systems are to truly become organisations with a memory, we need to expand how we understand - and put into practice- the processes of creating, maintaining and using collective memories. Remembrance, commemoration and memorialisation are wide-spread human activities, and are components of some of the most celebrated journeys of safety improvement in healthcare and elsewhere.[7,8,9] However, remembrance has largely been overlooked in the theory and practice of patient safety. Exploring the social practices of remembrance, and the complex relationships these practices have with learning and improvement, may reveal new ways of fostering the compassionate, reflective and just cultures that healthcare seeks to build. More fundamentally, it may help to shift research attention to the collective and emotional aspects of organisational memory that have received little attention to date. Remembrance therefore needs to be taken much more seriously, both in research and practice. 


\section{The future of the past}

Given how little is currently known, a systematic programme of research might begin by focusing on four key areas. First, what practical forms can remembrance take and in what ways do these differ according to institutional setting, type of event, nature of community or scale of organisation? Second, under what conditions might remembrance be transformed into bureaucratic box-ticking or ritualised blameavoidance, and how might these self-serving actions be prevented? Third, how might remembrance best serve those who have been harmed and in what ways could patients, families and staff benefit from coming together in remembrance[25]? Fourth, and most instrumentally, how can remembrance contribute to active learning, cultural change and practical improvement? Specifically, in what ways might remembrance foster more candid, compassionate and just cultures? How does remembrance shape processes of sensemaking and knowledge production in different professional groups? How might remembrance align with—or reveal gaps in—other memory-making activities, such as incident reporting, the telling of patient stories, morbidity and mortality meetings, safety investigations and public inquiries? And how might films and narratives be crafted that create emotional, meaningful and reflective moments for staff to engage with and learn from past harm?[8,26,27,28]. Healthcare organisations and researchers concerned with understanding and learning from adverse patient safety events should more purposefully engage with the issues and challenges raised by remembrance, both in theory and in practice. In doing so, we should strive to create an environment where those who have been harmed might forgive, and those who have the power to prevent harm can learn. 


\section{REFERENCES}

1Department of Health. An Organisation With a Memory: Report of an Expert Group on Learning from Adverse Events in the NHS Chaired by the Chief Medical Officer. London: Department of Health 2000.

2 Bosk CL. Forgive and Remember: Managing Medical Failure. Chicago: Chicago University Press 1979.

3. Sujan M. An organisation without a memory: A qualitative study of hospital staff perceptions on reporting and organisational learning for patient safety. Reliability Engineering and System Safety 2015;144, 45- 52.

4 Blustein J M. Forgiveness and Remembrance: Remembering Wrongdoing in Personal and Public Life. Oxford: Oxford University Press 2014.

5 Barsalou J , Baxter V. The Urge to Remember: The Role of Memorials in Social Reconstruction and Transitional J ustice; Stabilisation and Reconstruction Series 5. Washington, DC: United States Institute of Peace 2007.

6 Brett S, Bickford L, Sevenko L, Rios M. Memorialisation and Democracy: State Policy and Civic Action. New York: International Center for Transitional J ustice 2007.

7J apan Airlines Safety Promotion Center https:/ / www.jal.com/ en/ flight/ safety/ center/ Date accessed March 2016. 8 Virginia Mason Institute: Terrible tragedy and powerful legacy of preventable death http:// vminstitute.wpengine.com/ ?p=1051 Date accessed March 2016. 
9 Remembering NASA's heroes

http://www.nasa.gov/externalflash/DOR2016/index.html Date aocessed March 2016.

10 Titcombe J . J oshua's Story: Uncovering the Morecambe Bay NHS Scandal. London: Anderson Wallace 2015.

11 Studdert DM, Mello MM, Gawande AA, Brennan TA, Wang YC. Disclosure of medical injury to patients: an improbable risk management strategy. Health Aff 2007;26(1):215- 26.

12 Reason J. Managing the Risks of Organizational Accidents. Aldershot: Ashgate 1997.

13 Macrae C. Close Calls: Managing Risk and Resilience in Airline Flight Safety. London: Palgrave 2014.

14 Maitlis S, Christianson M. Sensemaking in Organizations: Taking Stock and Moving Forward. Acad Manag Ann. 2014;8(1):57- 125.

15 Vincent C, Amalberti R. Safer Healthcare: Strategies for the Real World. London: Springer 2016.

16 Card AJ , Klein VR. A new frontier in healthcare risk management: Working to reduce avoidable patient suffering. J ournal of Healthcare Risk Management, 2016;35(3):31- 37.

$17 \mathrm{Wu}$ AW, Steckelberg RC. Medical error, incident investigation and the second victim: doing better but feeling worse? BMJ Qual Saf 2012;21:267-270.

18 Heyhoe J, Birks Y, Harrison R, O'Hara J K. The role of emotion in patient safety: Are we brave enough to scratch beneath the surface? J R Soc Med 2015;109(2):5258. 
19 Heuer F, Reisberg D. Vivid memories of emotional events: The accuracy of remembered minutiae. Mem Cognit 1990;18(5):496- 50.

20 Maitlis S, Vogus TJ , Lawrence TB. Sensemaking and emotion in organizations. Organizational Psychology Review 2013;3(3):222-247.

21 Parkinson B, Simons G. Worry spreads: Interpersonal transfer of problem-related anxiety. Cogn Emot 2012;26(3):462-479.

22 Macrae C. Early warnings, weak signals and learning from healthcare disasters. BMJ Qual Saf 2014;23:440-445.

23 Hood C, Rothstein H, Baldwin R. The Government of Risk: Understanding Risk Regulation Regimes. Oxford: Oxford University Press 2001.

24 Dixon-Woods M. The Tombstone Effect: Long Shadows and the Pursuit of Comfort. In: Dixon R, Lodge M, eds. Explorations in Governance: A Collection of Papers in Honour of Christopher Hood. Oxford: Institute for Government 2012:3233.

25 Berlinger N. After Harm: Medical Error and the Ethics of Forgiveness. London: J ohns Hopkins University Press 2007.

26 Bromiley M. Have you ever made a mistake? RCoA Bulletin 2008;48:2442-2445. 27 Patient Stories https:// www.patientstories.org.uk Date accessed March 2016 28 The Human Factor: Learning from Gina's Story

https:// www.youtube.com/watch?v=IJ foLvLLoFo Date accessed March 2016 